\title{
Relationship between scintillation properties and crystallite sizes in $\mathrm{Y}_{2} \mathrm{O}_{3}: \mathrm{Eu}^{3+}$
}

\author{
Maike de Oliveira Krauser ${ }^{\mathrm{a}, \mathrm{b}}$, Higor Henrique de Souza Oliveira ${ }^{\mathrm{a}, \mathrm{c}}$, Marco Aurélio Cebim ${ }^{\mathrm{a}}$, \\ Marian Rosaly Davolos ${ }^{\mathrm{a}, *}$
}

a Universidade Estadual Paulista (Unesp), Instituto de Química, Araraquara, SP, Brazil

${ }^{\mathrm{b}}$ Universidade Federal do Tocantins (UFT), Química Ambiental, Gurupi, TO, Brazil

${ }^{\mathrm{c}}$ Instituto Federal de Educação, Ciência e Tecnologia de São Paulo (IFSP), Matão, SP, Brazil

\section{A R T I C L E IN F O}

\section{Keywords:}

Scintillation

Crystallite size

$\mathrm{Y}_{2} \mathrm{O}_{3}$

XEOL

\begin{abstract}
A B S T R A C T
This work describes the relation between crystallite size and scintillation properties of $\mathrm{Eu}^{3+}$-doped yttrium oxide. Nanocrystalline $\mathrm{Y}_{2} \mathrm{O}_{3}: \mathrm{Eu}^{3+} 1.0 \mathrm{~mol} \%$ powders were prepared by Pechini method and thermal treated at 700-1100 ${ }^{\circ} \mathrm{C}$. The thermal treatment temperature has influence on the crystallite sizes of $\mathrm{Y}_{2} \mathrm{O}_{3}: \mathrm{Eu}^{3+} \mathrm{nano}^{3}$ crystals and, consequently, on the structural and electronic defects concentration, which contributes to nonradiative recombination routes in scintillation materials. This relationship between the crystallite sizes and scintillation properties was investigated by X-ray diffraction and X-ray excited optical luminescence measurements.
\end{abstract}

\section{Introduction}

In the two past decades, the preparation and characterization of rare earth sesquioxide nanoparticles have been the subject of several studies [1-3]. Referent to bulk materials, nanometric structures may present different spectroscopic properties, which are tightly bounded to crystalline structure and particle morphology $[4,5]$.

Yttrium sesquioxide can be used as a suitable inorganic host for doping with trivalent lanthanides $\left(\mathrm{Ln}^{3+}\right)$, since this process is an isovalent substitution $\left(\mathrm{Y}^{3+} \rightarrow \mathrm{Ln}^{3+}\right)$, in which the ionic radii difference is not pronounced and both elements present similar electronegativities [6]. Furthermore, trivalent yttrium compounds at room temperature do not present luminescence (bands or lines) in the visible region of the electromagnetic spectrum [7].

Rare earths have been used to prepare optical materials and one of the interest applications is on scintillation field $[8,9]$. These materials are applied in radiation detection and nuclear spectroscopy [10], medical imaging [11] and other properties based on ionizing radiation detection [12].

In this way, $\mathrm{Y}_{2} \mathrm{O}_{3}$ can be used in the preparation of several doped compounds with different optical properties. Doping with different trivalent lanthanide cations leads to materials with different luminescent properties: for instance, the $\mathrm{Tb}^{3+}$ as a dopant into a $\mathrm{Y}_{2} \mathrm{O}_{3}$ matrix results in a green light phosphor [5], while the doping with $\mathrm{Yb}^{3+}$ can produce a luminescence upconversion material $[13,14]$.
Several methods are applied in order to obtain nanocrystals of $\mathrm{Y}_{2} \mathrm{O}_{3}$ and $\mathrm{Y}_{2} \mathrm{O}_{3}: \mathrm{Ln}^{3+}$, such as microemulsion [15], spray pyrolysis [4], polymeric precursors [16], aerosol-UV [14], homogeneous precipitation in aqueous $[17,18]$ and non-aqueous solution [3]. $\mathrm{Eu}^{3+}$-doped $\mathrm{Y}_{2} \mathrm{O}_{3}$ compounds are extensively studied and their spectroscopic properties are related to intraconfigurational $4 f^{6} \rightarrow 4 f^{6}$ transitions of the $\mathrm{Eu}^{3+}$ ions. Specifically, the emission lines at wavelength range from 550 to $800 \mathrm{~nm}$ for $\mathrm{Y}_{2} \mathrm{O}_{3}: \mathrm{Eu}^{3+}$ materials are assigned to ${ }^{5} \mathrm{D}_{0} \rightarrow{ }^{7} \mathrm{~F}_{\mathrm{J}}(\mathrm{J}=$ $0,1,2,3,4)$ transitions, and these sets of lines can be used as a structural probe to study the chemical environment around $\mathrm{Eu}^{3+}$ ions [7].

In this work, we present studies on yttrium oxide, $\mathrm{Y}_{2} \mathrm{O}_{3}$, doped with $\mathrm{Eu}^{3+} 1.0 \mathrm{~mol} \%$ ions. The influence of the crystallite sizes on the scintillation properties was investigated by X-ray diffraction and X-ray excited optical luminescence measurements.

\section{Experimental section}

\subsection{Synthesis of $\mathrm{Y}_{2} \mathrm{O}_{3}: \mathrm{Eu}^{3+} 1.0 \mathrm{~mol} \%$ particles}

$\mathrm{Y}_{2} \mathrm{O}_{3}: \mathrm{Eu}^{3+} 1.0 \mathrm{~mol} \%$ was prepared by the Pechini method (polymeric precursors method) [19], using $\mathrm{Y}_{2} \mathrm{O}_{3}$ and $\mathrm{Eu}_{2} \mathrm{O}_{3}$ (Sigma-Aldrich, 99.999\%) as starter reagents. All other used reagents were analytical grade. In this method, $\mathrm{Y}\left(\mathrm{NO}_{3}\right)_{3}$ and $\mathrm{Eu}\left(\mathrm{NO}_{3}\right)_{3}$ aqueous solutions at defined proportions were added in a beaker containing citric acid (CA) in 1:3 (metal:CA) molar ratio. The $\mathrm{pH}$ of the resulting solution was

\footnotetext{
* Corresponding author.

E-mail address: davolos@iq.unesp.br (M.R. Davolos).
} 
adjusted to 5.0 with $0.1 \mathrm{~mol} \mathrm{~L}^{-1} \mathrm{NH}_{4} \mathrm{OH}$ solution, and this system was stirred for $1 \mathrm{~h}$. After this period, ethylene glycol (EG) was added to the solution at a 1:16 (metal:EG) molar ratio, and heated $\left(\sim 100^{\circ} \mathrm{C}\right)$ until the formation of a yellowish polymeric resin, which was thermally treated in a tubular furnace with static air atmosphere for $4 \mathrm{~h}$ from $700^{\circ}$ to $1100{ }^{\circ} \mathrm{C}$, temperature above the rare earth oxides phase formation, using polymeric precursors, temperature, that is, about $650{ }^{\circ} \mathrm{C}$ [20-23].

\subsection{Characterization techniques}

The samples were characterized by powder X-ray diffraction (XRD) using copper $\mathrm{K}_{\alpha}$ radiation in a Rigaku Rint 2000/PC diffractometer operating at $42 \mathrm{kV}$ and $120 \mathrm{~mA}$. The data were collected in the $2 \theta / \theta$ mode in the $15-60^{\circ}$ range with a $0.02 / 2 \mathrm{~s}$ propagation step. Particle shape and size were analyzed in a field emission gun scanning electron microscope with field emission gun (FEG-SEM) Jeol JSM 7500F operating at $2 \mathrm{kV}$ with a detector of secondary electrons. Fourier transform infrared spectroscopy (FT-IR) measurements were performed in the $400-4000 \mathrm{~cm}^{-1}$ wavenumber window, using sample/KBr pellets, in a Perkin Elmer Spectrum 2000 spectrometer. To evaluate the luminescent properties, excitation and emission spectra were recorded by photoluminescence spectroscopy (PLS), performed in SPEX Fluorolog 2 fluorimeter, with $450 \mathrm{~W}$ xenon lamp, double monochromator and Hammamatsu photomultiplier. The scintillation properties were investigated by X-ray excited optical luminescence (XEOL) measurements performed by copper soft X-ray excitation from Rigaku X-ray source and the light emission was detected with a iDus DV401A-BV CCD camera coupled to a SHAMROCK 163 spectrometer, Andor Tech [24].

\section{Results and discussion}

\subsection{Structure}

The FT-IR spectra, Fig. 1, show peaks of M-O bond below $650 \mathrm{~cm}^{-1}$. Comparing with $\mathrm{M}-\mathrm{O}$ bond, the relative intensities of $\mathrm{H}-\mathrm{O}-\mathrm{H}$ bending, $\mathrm{C}-\mathrm{O}$ symmetrical, ant-symmetrical stretching from $\mathrm{CO}_{3}{ }^{-2}$ at a $1500 \mathrm{~cm}^{-1}$ and $\mathrm{O}-\mathrm{H}$ on $3500 \mathrm{~cm}^{-1}$ decrease as a function of thermal treatment temperature. Residues of these species at $1100{ }^{\circ} \mathrm{C}$ may be related to moisture and surface carbonation.

Fig. 2 shows the XRD results, indicating the formation of crystalline products, in accord of JCPDS 41-1105 yttrium oxide $\left(\mathrm{Y}_{2} \mathrm{O}_{3}\right)$ pattern. This rare earth compound belongs to the cubic crystal system with the Ia-3 space group (No. 206, $\mathrm{Z}=16$ ). The $\mathrm{Y}^{3+}$ are coordinated by six oxygens in two different sites, $\mathrm{S}_{6}$ and $\mathrm{C}_{2}$ [25]. Scherrer crystallite sizes (D) obtained from Eq. (1) were evaluate for all samples using the (222) diffraction.

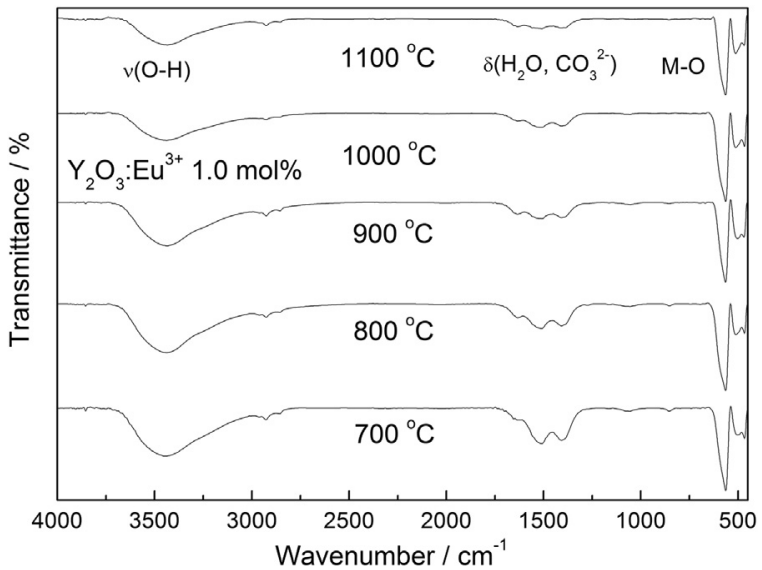

Fig. 1. FT-IR spectra from $\mathrm{Y}_{2} \mathrm{O}_{3}: \mathrm{Eu}^{3+} 1.0 \mathrm{~mol} \%$ prepared at different temperatures.

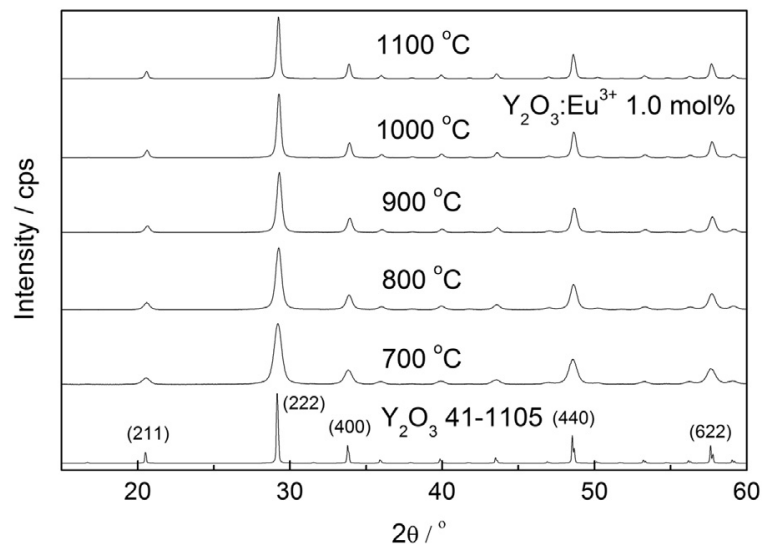

Fig. 2. $\mathrm{XRD}$ patterns of $\mathrm{Y}_{2} \mathrm{O}_{3}: \mathrm{Eu}^{3+} 1.0 \mathrm{~mol} \%$ prepared at different temperatures.

$D=\frac{K \lambda}{\sqrt{\left(\beta_{h k l}\right)_{\text {measured }}^{2}-(\beta)^{2}{ }_{\text {instrumental }}} \cos \theta_{h k l}}$,

where $\lambda$ is the X-ray wavelength (1.5406 $\AA$ ), $\beta$ is the full width at half maximum (FWHM), $\theta_{h k l}$ the Bragg angle of the $h k l$ peak, and $K$ is a shape constant equal to 0.89 .

Crystallite sizes as a function of thermal treatment temperature are presented in Fig. 3. A nearly linear enlargement of crystallite sizes as a function of the temperature is found for this system, with crystallite sizes ranged from 15 to $45 \mathrm{~nm}$. According to the literature [26-29], the decreasing of crystallite sizes can have raised the quantity of defects, which is associated with the positive pressure of formation inside the crystallites. This can contributes to the formation of oxygen vacancies, along with interstitial species, providing structure mismatches in relation to ideal single crystal compounds.

Fig. 4 shows FEG-SEM images of $\mathrm{Y}_{2} \mathrm{O}_{3}: \mathrm{Eu}^{3+} 1.0 \mathrm{~mol} \%$ sample thermal treated at $1100{ }^{\circ} \mathrm{C}$. The FEG-SEM images show micrometrics particles formed due to the sintering process. These microparticles (Fig. 4A) are formed by nanoparticles aggregated, that can be distinguished in Fig. 4B, morphology usually obtained for rare earth oxides using the Pechini method $[20,21]$.

\subsection{Luminescence properties}

Excitation and emission spectra of the $\mathrm{Y}_{2} \mathrm{O}_{3}: \mathrm{Eu}^{3+} 1.0 \mathrm{~mol} \%$ samples are shown in the Fig. 5. The excitation spectra do not show changes with respect to the heat treatment temperature and show charge

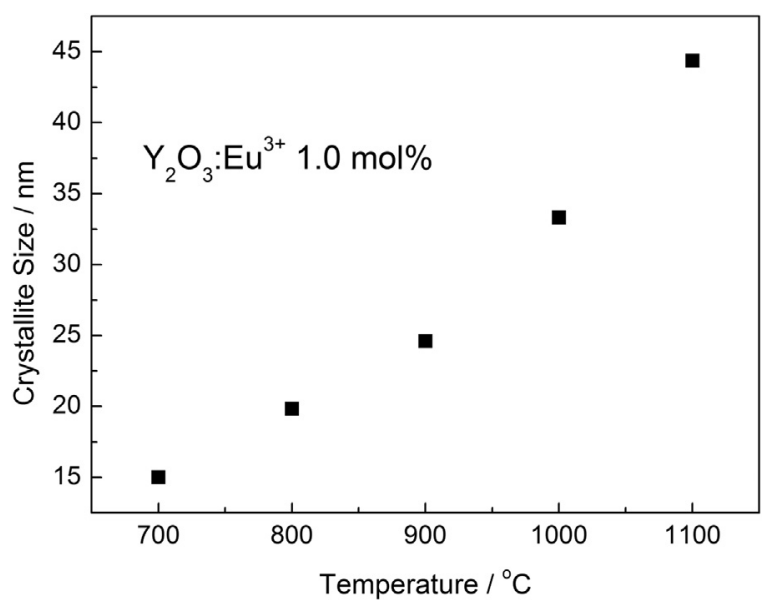

Fig. 3. Crystallite size, calculated using Scherrer equation, of $\mathrm{Y}_{2} \mathrm{O}_{3}: \mathrm{Eu}^{3+} 1.0$ mol\% as a function of thermal treatment temperature. 


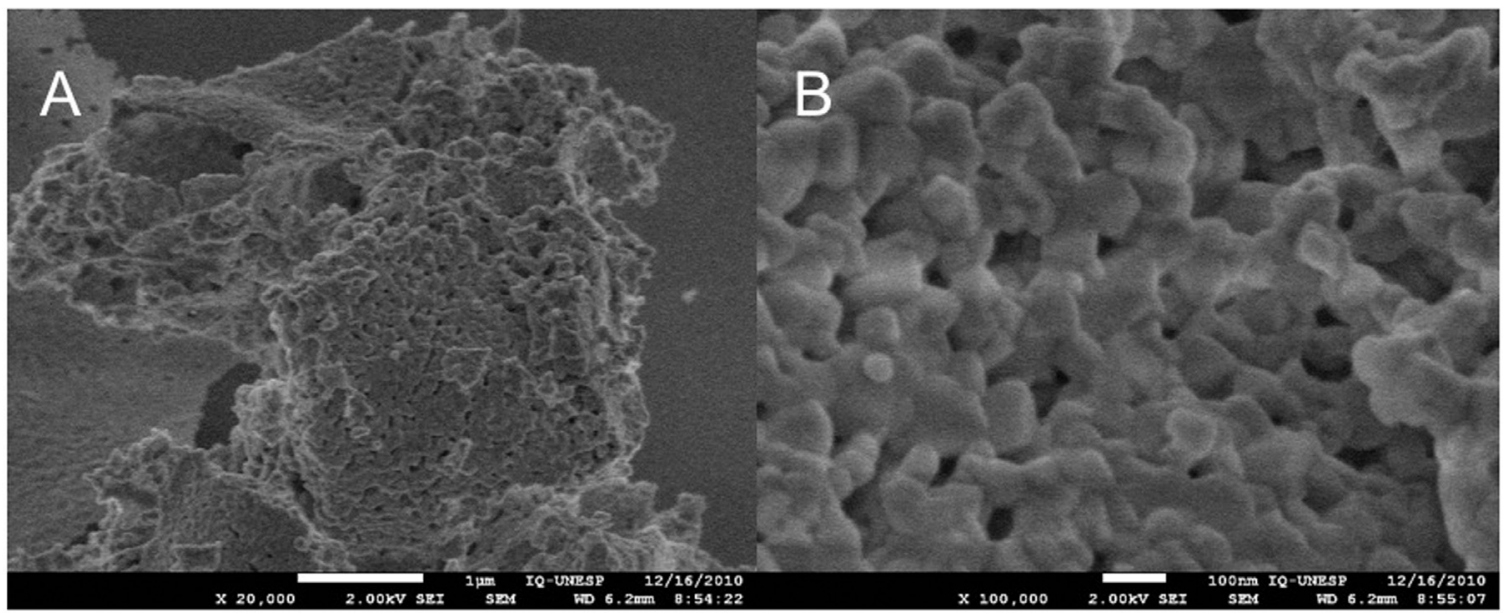

Fig. 4. FEG-SEM of $\mathrm{Y}_{2} \mathrm{O}_{3}: \mathrm{Eu}^{3+} 1.0 \mathrm{~mol} \%$ sample thermal treated at $1100{ }^{\circ} \mathrm{C}$.

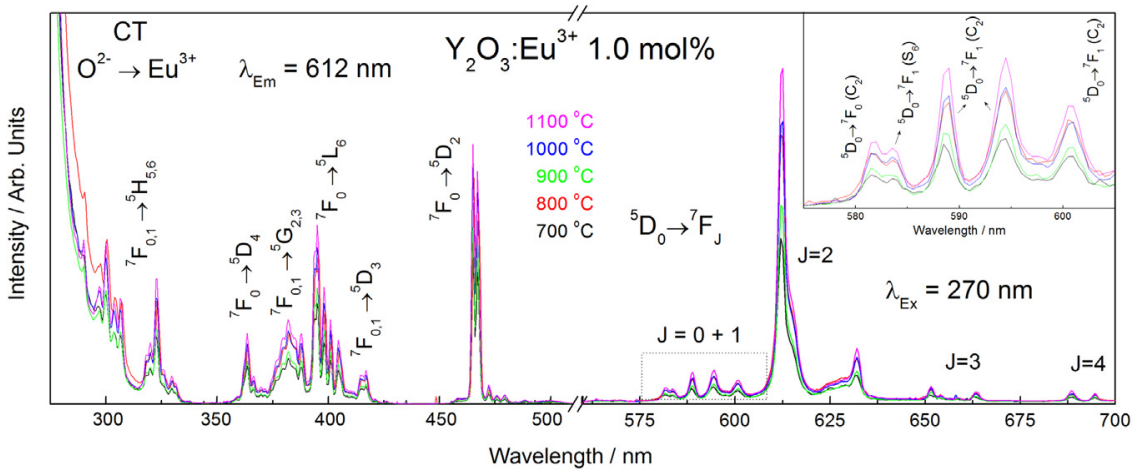

Fig. 5. Excitation and emission spectra of $\mathrm{Y}_{2} \mathrm{O}_{3}: \mathrm{Eu}^{3+} 1.0 \mathrm{~mol} \%$ prepared at $700-1100{ }^{\circ} \mathrm{C}, \lambda_{\mathrm{Em}}=612 \mathrm{~nm} \lambda_{\mathrm{Ex}}=270 \mathrm{~nm}$.

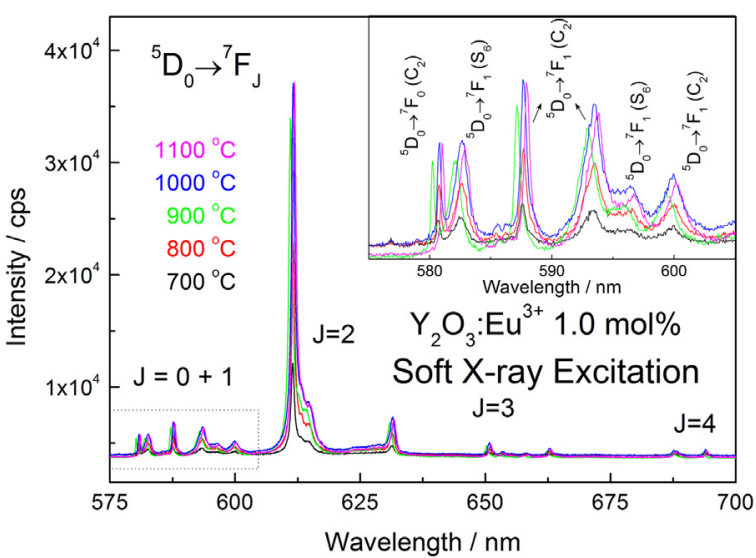

Fig. 6. Emission spectra of $\mathrm{Y}_{2} \mathrm{O}_{3}: \mathrm{Eu}^{3+} 1.0 \mathrm{~mol} \%$ prepared at $700-1100{ }^{\circ} \mathrm{C}$ performed under soft $\mathrm{X}$-ray excitation $\left(\mathrm{Cu} \mathrm{K}_{\alpha}\right)$ with voltage of $35 \mathrm{kV}$ and current of $20 \mathrm{~mA}$

transfer band (CT) below $300 \mathrm{~nm}$ and expected f-f transitions for the ions $\mathrm{Eu}^{3+}{ }^{7} \mathrm{~F}_{0} \rightarrow{ }^{5} \mathrm{D}_{2},{ }^{7} \mathrm{~F}_{0,1} \rightarrow{ }^{5} \mathrm{D}_{3},{ }^{7} \mathrm{~F}_{0} \rightarrow{ }^{5} \mathrm{~L}_{6},{ }^{7} \mathrm{~F}_{0,1} \rightarrow{ }^{5} \mathrm{G}_{2,3},{ }^{7} \mathrm{~F}_{0} \rightarrow{ }^{5} \mathrm{D}_{4}$ and ${ }^{7} \mathrm{~F}_{0,1} \rightarrow{ }^{5} \mathrm{H}_{5,6}$ in order of energy. The emission spectral profiles are the same for all samples. The spectra show the characteristic emission lines of the $\mathrm{Eu}^{3+}$ ions, assigned to ${ }^{5} \mathrm{D}_{0} \rightarrow{ }^{7} \mathrm{~F}_{\mathrm{J}}$ transitions, with $\mathrm{J}$ varying from 0 to 4. In yttrium oxide, $\mathrm{Eu}^{3+}$ ions occupy two different symmetry sites, being one $\left(\mathrm{C}_{2}\right)$ of low symmetry and other $\left(\mathrm{S}_{6}\right)$ of high symmetry [25]. Almost $75 \%$ of $\mathrm{Eu}^{3+}$ ions are present at $\mathrm{C}_{2}$ sites, resulting in more intense ${ }^{5} \mathrm{D}_{0} \rightarrow{ }^{7} \mathrm{~F}_{2}$ emission lines than ${ }^{5} \mathrm{D}_{0} \rightarrow{ }^{7} \mathrm{~F}_{1}$ [25]. Higher thermal treatment temperature results in emission intensity increase, because the gain on crystallinity and the growth the crystallite size can reduced

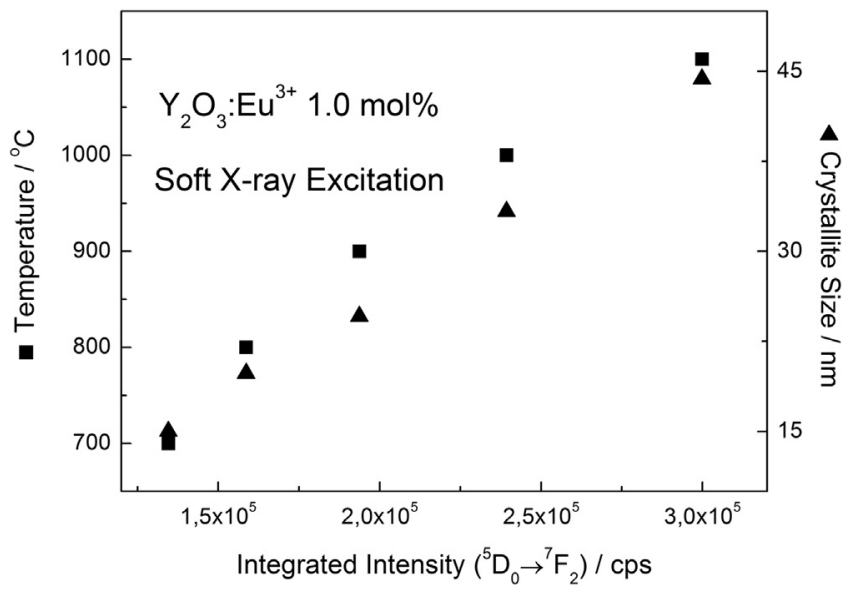

Fig. 7. Relation among thermal treatment temperature, crystallite size and integrated emission intensity from ${ }^{5} \mathrm{D}_{0} \rightarrow{ }^{7} \mathrm{~F}_{2}$ transition of $\mathrm{Y}_{2} \mathrm{O}_{3}: \mathrm{Eu}^{3+} 1.0 \mathrm{~mol} \%$.

nonradiative recombination rates [30].

The X-ray-excited luminescence was registered with soft X-ray excitation $\left(\mathrm{Cu} \mathrm{K}_{\alpha}\right)$ source operating at $35 \mathrm{kV}$ and $20 \mathrm{~mA}$. Fig. 6 shows the emission spectra from $\mathrm{Y}_{2} \mathrm{O}_{3}: \mathrm{Eu}^{3+}$, which present the emission lines of $\mathrm{Eu}^{3+}$ ion assigned to ${ }^{5} \mathrm{D}_{0} \rightarrow{ }^{7} \mathrm{~F}_{\mathrm{J}}$, with the highest intensity from ${ }^{5} \mathrm{D}_{0} \rightarrow{ }^{7} \mathrm{~F}_{2}$ transition, the same emission profile observed in photoluminescence. Taking into account that X-ray excitation luminescence measurements were obtained to minimize package effects and consequently scattered luminescence intensity variations, the integrated emission intensity from ${ }^{5} \mathrm{D}_{0} \rightarrow{ }^{7} \mathrm{~F}_{2}$ transition was calculated and compared in a function of the preparation temperature. The Fig. 7 shows an almost linear 


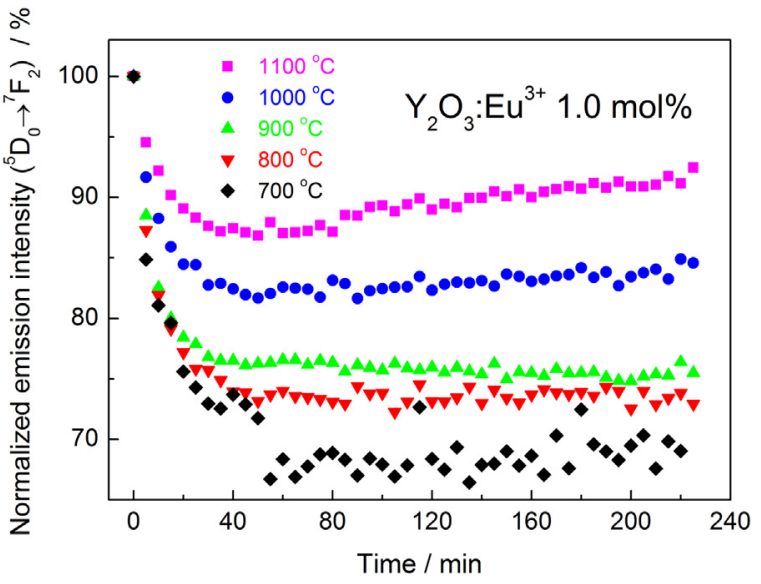

Fig. 8. Normalized integrated emission intensity from ${ }^{5} \mathrm{D}_{0} \rightarrow{ }^{7} \mathrm{~F}_{2}$ of $\mathrm{Y}_{2} \mathrm{O}_{3}: \mathrm{Eu}^{3+}$ $1.0 \mathrm{~mol} \%$ as a function of time under soft X-ray excitation.

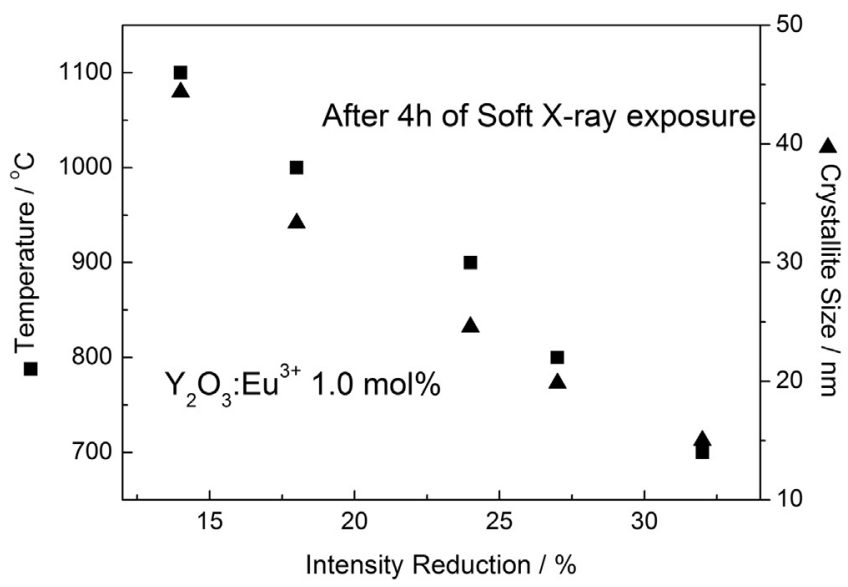

Fig. 9. Relation among thermal treatment temperature, crystallite size and ${ }^{5} \mathrm{D}_{0} \rightarrow{ }^{7} \mathrm{~F}_{2}$ transition intensity reduction of $\mathrm{Y}_{2} \mathrm{O}_{3}: \mathrm{Eu}^{3+} 1.0 \mathrm{~mol} \%$ after $4 \mathrm{~h}$ soft Xray exposure.

increasing of the integrated emission intensity from ${ }^{5} \mathrm{D}_{0} \rightarrow{ }^{7} \mathrm{~F}_{2}$ transition with the preparation temperature and consequently, with the crystallite sizes. Firstly, considering that increasing the sample thermal treatment temperature, the crystallite sizes increase and consequently its crystallinity, and secondly, that pair recombination occurs by nonradiative pathways and that particularly does not directly involve luminescent centers, this increase of the emission intensity suggests nonradiative rates decrease as a function of crystallinity.

The Fig. 8 shows the integrated emission intensity from ${ }^{5} \mathrm{D}_{0} \rightarrow{ }^{7} \mathrm{~F}_{2}$ transition as a function of irradiation time up to $240 \mathrm{~min}$ of soft X-ray exposure. During the irradiation on the first $40 \mathrm{~min}$, it is possible to evidence the formation of defects in the samples, which results in decreasing of luminescence because it is generated more nonradiative routes for the recombination of electron and holes before they can recombine radiatively $[8,12]$. After this first step, the formation of new defects and the capacity of recombination stay balanced until the end of measurement. In the more crystalline samples, it is possible to observe the probability of radiative recombination on the activator centers increases that, in this case, results in the emission of $\mathrm{Eu}^{3+}$ ions.

Integrated emission intensity decreasing observed during the measurement was not the same for all samples. Fig. 9 shows the relationship of the temperature or the crystallite size as a function of the ${ }^{5} \mathrm{D}_{0} \rightarrow{ }^{7} \mathrm{~F}_{2}$ transition intensity decreasing. More crystalline samples, that shows larger crystallite size, present less emission intensity reduction. The sample prepared at $1100^{\circ} \mathrm{C}$, which presents $45 \mathrm{~nm}$ crystallite size, shows $12 \%$ of emission intensity reduction, however the sample treated at $700{ }^{\circ} \mathrm{C}$ with $15 \mathrm{~nm}$ crystallite size, shows $34 \%$. Also emission intensity is very sensitive to defects concentration. In this case, higher thermal treatment temperature results in materials with less defects concentration on the particles, that probably increases the scintillator efficiencies.

\section{Conclusion}

$\mathrm{Y}_{2} \mathrm{O}_{3}: \mathrm{Eu}^{3+} 1.0 \mathrm{~mol} \%$ can be easily synthesized by the Pechini method. The samples present high crystalline phase and aggregates of nanoparticles with variation of crystallite size from 15 to $45 \mathrm{~nm}$, depending on the thermal treatment temperature.

All the samples show the emission from ${ }^{5} \mathrm{D}_{0} \rightarrow{ }^{7} \mathrm{~F}_{\mathrm{J}}(J=0,1,2,3$ e 4) transitions of $\mathrm{Eu}^{3+}$ ions. The emission intensity presents high dependency of the thermal treatment temperature or crystallite size, mainly in the scintillation properties when the emission process is strongly dependent of the number of defects in the material. In the $\mathrm{Y}_{2} \mathrm{O}_{3}: \mathrm{Eu}^{3+} 1.0 \mathrm{~mol} \%$ obtained by Pechini method, the sample prepared at $1100{ }^{\circ} \mathrm{C}$ presents $45 \mathrm{~nm}$ crystallite size and exhibits the best scintillation properties.

\section{Acknowledgments}

The authors are grateful to FAPESP, CNPq and CAPES (Brazilian Agencies) for the financial support. This work was developed with institutional infrastructure financed by FAPESP, CAPES and CNPq (Brazilian Agencies), as well as from CNPq grant number 312482/20132.

\section{References}

[1] H.S. Yoo, H.S. Jang, W.B. Im, J.H. Kang, D.Y. Jeon, Particle size control of a monodisperse spherical Y2O3:Eu3 + phosphor and its photoluminescence properties, J. Mater. Res. 22 (2011) 2017-2024.

[2] N. Vu, T. Kim Anh, G.-C. Yi, W. Strek, Photoluminescence and cathodoluminescence properties of Y2O3:Eu nanophosphors prepared by combustion synthesis, J. Lumin. 122-123 (2007) 776-779.

[3] Y. Sun, L. Qi, M. Lee, B.I. Lee, W.D. Samuels, G.J. Exarhos, Photoluminescent properties of Y2O3:Eu3 + phosphors prepared via urea precipitation in non-aqueous solution, J. Lumin. 109 (2004) 85-91.

[4] N. Joffin, J. Dexpert-Ghys, M. Verelst, G. Baret, A. Garcia, The influence of microstructure on luminescent properties of:Eu prepared by spray pyrolysis, J. Lumin. 113 (2005) 249-257.

[5] H. Song, J. Wang, Dependence of photoluminescent properties of cubic Y2O3:Tb3+ nanocrystals on particle size and temperature, J. Lumin. 118 (2006) 220-226.

[6] R.D. Shannon, Revised effective ionic radii and systematic studies of interatomic distances in halides and chalcogenides, Acta Crystallogr. Sect. A 32 (1976) 751-767.

[7] G. Blasse, B.C. Grabmaier, Luminescent Materials, 1st ed., Springer-Verlag Telos, New York, 1994.

[8] G. Blasse, Scintillator materials, Chem. Mater. 6 (1994) 1465-1475.

[9] P. Dorenbos, The $5 \mathrm{~d}$ level positions of the trivalent lanthanides in inorganic compounds, J. Lumin. 91 (2000) 155-176.

[10] E.A. McKigney, R.E. Del Sesto, L.G. Jacobsohn, Pa Santi, R.E. Muenchausen, K.C. Ott, T. Mark McCleskey, B.L. Bennett, J.F. Smith, D. Wayne Cooke, Nanocomposite scintillators for radiation detection and nuclear spectroscopy, Nucl. Instrum. Methods Phys. Res. Sect. A: Accel. Spectrom. Detect. Assoc. Equip. 579 (2007) 15-18.

[11] P. Lecoq, Development of new scintillators for medical applications, Nucl. Instrum. Methods Phys. Res. Sect. A: Accel. Spectrom. Detect. Assoc. Equip. 809 (2015) 130-139.

[12] S.E. Derenzo, M.J. Weber, E. Bourret-Courchesne, M.K. Klintenberg, The quest for the ideal inorganic scintillator, Nucl. Instrum. Methods Phys. Res. Sect. A: Accel. Spectrom. Detect. Assoc. Equip. 505 (2003) 111-117.

[13] A.M. Pires, O.A. Serra, M.R. Davolos, Morphological and luminescent studies on nanosized Er, Yb-Yttrium oxide up-converter prepared from different precursors, J Lumin. 113 (2005) 174-182.

[14] R. Salhi, J.-L. Deschanvres, Efficient upconversion in Er3 + doped Y2O3/Si thin film deposited by aerosol UV-assisted MOCVD process, J. Lumin. 170 (2016) 231-239.

[15] X. Wu, Y. Liang, R. Liu, Y. Li, The photoluminescence properties of Y2O3:Eu3 + prepared by surfactant assisted co-precipitation-molten salt synthesis, Mater. Res. Bull. 45 (2010) 594-597.

[16] J.L. Ferrari, A.M. Pires, M.R. Davolos, The effect of Eu3 + concentration on the Y2O3 host lattice obtained from citrate precursors, Mater. Chem. Phys. 113 (2009) 587-590.

[17] A.M. Pires, M.R. Davolos, E.B. Stucchi, Eu3 + as a spectroscopic probe in phosphors 
based on spherical fine particle gadolinium compounds q, Int. J. Inorg. Mater. 3 (2001) 785-790.

[18] A.M. Pires, M.R. Davolos, C.O. Paiva-Santos, E.B. Stucchi, J. Flor, New X-ray powder diffraction data and Rietveld refinement for Gd2O3 monodispersed fine spherical particles, J. Solid State Chem. 171 (2003) 420-423.

[19] M.P. Pechini, Method of preparing lead and alkaline earth titanates and niobates and coating method using the same to form capacitor, in, US3330697, 1967.

[20] H. Guo, M. Yin, N. Dong, M. Xu, L. Lou, W. Zhang, Effect of heat-treatment temperature on the luminescent properties of Lu2O3:Eu film prepared by Pechini sol-gel method, Appl. Surf. Sci. 243 (2005) 245-250.

[21] M. Galceran, M.C. Pujol, M. Aguiló, F. Díaz, Synthesis and characterization of nanocrystalline Yb:Lu2O3 by modified Pechini method, Mater. Sci. Eng.: B 146 (2008) 7-15.

[22] P. Yu, K. Zhang, H. Huang, M. Wen, Q. Li, W. Zhang, C. Hu, W. Zheng, Oxygen vacancies dependent phase transition of Y2O3 films, Appl. Surf. Sci. 410 (2017) $470-478$.

[23] J. Chen, B. Huang, C. Huang, X. Sun, Preparation of nanoscaled yttrium oxide by citrate precipitation method, J. Rare Earths 35 (2017) 79-84.

[24] M.A. Cebim, H.Hd.S. Oliveira, N. Barelli, M.R. Davolos, Sistema para realização de medidas de luminescência com excitação por Raios X, Quím. Nova 34 (2011) 1057-1062.

[25] M. Buijs, A. Meyerink, G. Blasse, Energy transfer between Eu3 + ions in a lattice with two different crystallographic sites: Y2O3:Eu3 +, Gd2O3:Eu3 + and Eu2O3, J. Lumin. 37 (1987) 9-20.

[26] T. Grzyb, M. Węcławiak, S. Lis, Influence of nanocrystals size on the structural and luminescent properties of GdOF:Eu3 +, J. Alloy. Compd. 539 (2012) 82-89.

[27] C.R. Stanek, M.R. Levy, K.J. McClellan, B.P. Uberuaga, R.W. Grimes, Defect identification and compensation in rare earth oxide scintillators, Nucl. Instrum. Methods Phys. Res. Sect. B: Beam Interact. Mater. At. 266 (2008) 2657-2664.

[28] M.R. Levy, C.R. Stanek, A. Chroneos, R.W. Grimes, Defect chemistry of doped bixbyite oxides, Solid State Sci. 9 (2007) 588-593.

[29] R. Srinivasan, N.R. Yogamalar, J. Elanchezhiyan, R.J. Joseyphus, A.C. Bose, Structural and optical properties of europium doped yttrium oxide nanoparticles for phosphor applications, J. Alloy. Compd. 496 (2010) 472-477.

[30] D.J. Robbins, B. Cockayne, J.L. Glasper, B. Lent, The temperature dependence of rare-earth activated garnet phosphors, J. Electrochem. Soc.: Solid-State Sci. Technol. 126 (1979) 1213-1220. 